\title{
Tree Growth Modelling Constrained by Growth Equations
}

\author{
Lei $\mathrm{Yi}^{1,2,3}$, Hongjun $\mathrm{Li}^{3}$, Jianwei Guo ${ }^{2, *}$, Oliver Deussen ${ }^{4,5}$ and Xiaopeng Zhang ${ }^{2, *}$ \\ ${ }^{1}$ School of Computer Science, Northwestern Polytechnical University, Xi'an, China \\ lei.yi@nlpr.ia.ac.cn \\ ${ }^{2}$ NLPR-LIAMA, Institute of Automation, CAS, Beijing, China \\ ${ }^{3}$ College of Science, Beijing Forestry University, Beijing, China \\ lihongjun69@bjfu.edu.cn \\ ${ }^{4}$ University of Konstanz, Konstanz, Germany \\ oliver.deussen@uni-konstanz.de \\ ${ }^{5}$ VCC, Shenzhen Institute of Advanced Technology (SIAT), CAS, China
}

\begin{abstract}
Modelling and simulation of tree growth that is faithful to the living environment and numerically consistent to botanic knowledge are important topics for realistic modelling in computer graphics. The realism factors concerned include the effects of complex environment on tree growth and the reliability of the simulation in botanical research, such as horticulture and agriculture. This paper proposes a new approach, namely, integrated growth modelling, to model virtual trees and simulate their growth by enforcing constraints of environmental resources and tree morphological properties. Morphological properties are integrated into a growth equation with different parameters specified in the simulation, including its sensitivity to light, allocation and usage of received resources and effects on its environment. The growth equation guarantees that the simulation procedure numerically matches the natural growth phenomenon of trees. With this technique, the growth procedures of diverse and realistic trees can also be modelled in different environments, such as resource competition among multiple trees.
\end{abstract}

Keywords: tree modelling, integrated growth modelling, growth equations

\section{Introduction}

Tree growth modelling with reliable shapes is essential for many applications, such as agriculture, forestry, landscape design and urban modelling. Instead of merely constructing a visually pleasing model, generating trees with a higher level of realism is important in tree modelling. Therefore, modelling should be performed based on biological and physical constraints to generate trees that have a direct representation of their shapes and growth process.

Plants usually grow under complex environmental impacts, and thus considering their biological mechanisms is necessary. Conversely, geometric models that satisfy growth equations are also

${ }^{*}$ Corresponding author: Jianwei Guo and Xiaopeng Zhang. (jianwei. guo@nlpr.ia.ac.cn, xpzhang@nlpr.ia.ac.cn) proved useful for other academic research. Tree growth equation can be used to numerically estimate the annual benefits for rain fall interception, pollutant uptake and carbon dioxide sequestration [LL05].

Plant growth simulation has been analysed in several references. Tree growth was simulated in [LK05] based on the L-system, which considers the effects of the environment on growth. Space colonization was proposed as a strategy in [RLP07] to model the skeleton structure by comprehensively considering the effects from environmental and between-branch spaces. Self-organization tree modelling (SOTM) was proposed in [PHL*09] as a modelling technique for simulating resource distribution, while considering the environmental effects. Light-guided tree modelling (LGTM) was proposed in [YLG*15] using environmental information. It suggests a new way to calculate the optimal shooting directions of branches based on light intensity. 


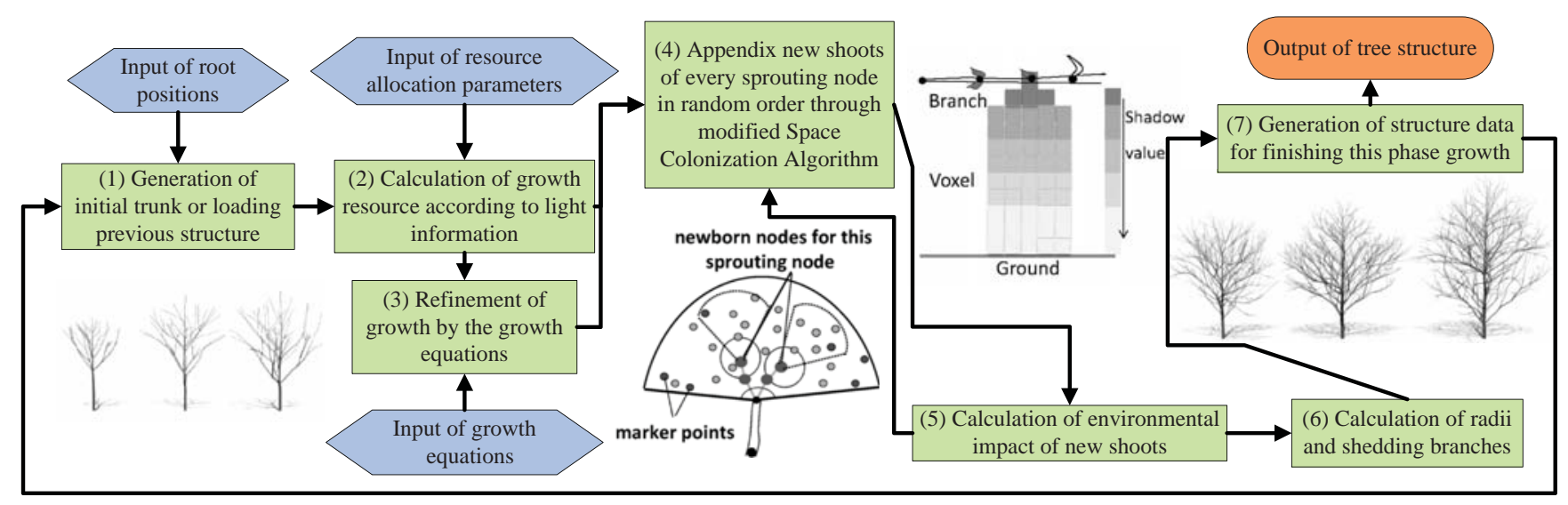

Figure 1: Overview of the algorithm for tree growth modelling constrained by growth equations.

However, some factors are worth considering when simulating the process of tree growth:

- Numerical analysis is a credible way to verify tree models rather than just using the human eyes. The growth process was also proven to satisfy some formulas or equations numerically [Zei93].

- Based on numerical models, the growth process should stop automatically. State-of-the-art methods use an iteration time to stop tree growth, which does not comply with tree height theory [KSJD04, Poo99] and natural tree attributes.

- The reaction of plants to the environment is altered according to the different growth stages.

In this paper, we propose a new approach, namely, integrated growth modelling (IGM), to simulate tree growth with constraints from tree properties and the environment, including light resource, space occupation and environmental impacts. The information is integrated into the growth equation using a small number of parameters. Meanwhile, light influence on growth is also reconsidered in plant growth condition. Therefore, our method addresses those issues listed in the preceding paragraph as well as presents a new tree growth mechanism by comprehensively considering the roles from external environmental factors and internal growth constraints.

The flow diagram of our algorithm is shown in Figure 1. This diagram illustrates how growth equations within the resource allocation model and environmental impacts can be integrated into the modelling procedure to simulate growth.

\section{Related Works}

Plant modelling has been a widespread concern over the last decades. A large number of studies have concentrated on tree modelling methods, where the main developments have been generally described in key references, including [dREF*88, WP95, DL06]. Generally speaking, plant modelling techniques are classified into three categories: static plant modelling without the growth process, procedural modelling and modelling constrained by environmental factors.

\subsection{Static plant modelling}

Static approaches are about getting the static plant shape, where the growth process is not emphasized. These methods differ from one another according to input data, e.g. images or 3D point clouds.

Image-based methods An image-based method was proposed in [QTZ*06] to model skeletons and reconstruct leaves from images, and provide a user-friendly editor for branch structure guided by $2 \mathrm{D}$ and $3 \mathrm{D}$ information. By using a statistical model to learn the leaf shape and appearance from images, the technique in [BNB13] can reconstruct and synthesize model leaves. A simple and effective method was developed in [LDY10] to compute an optimal 3D skeleton tree from estimated 2D/3D tree topology from images. Different from this method, Neubert et al. [NFD07] applied a particle system [RCSL03] to reconstruct models from images, which is another way to reconstruct tree skeletons.

Scan-based methods Acquiring 3D data from large objects, such as trees, is feasible and convenient through scanning devices. Based on a unilateral scanning point cloud, $\mathrm{Xu}$ et al. [XGC07] reconstructed a tree skeleton by building a $k$-nearest graph and minimum spanning tree to extract data, and then adding twigs by Lsystem. [LYO*10, LPC*11] proposed alternative ways to optimize and approximate shapes by branch patterns globally. Zhang et al. [ZLD*14] developed a data-driven method to synthesize trees by multiple-layer representation of scanned data.

Sketch-based methods Sketch modelling methods are often used to enhance reconstruction effects or compensate for missing data. The interactive methods in [TFX*08, WBCG09, CNX*08, IOI06, OOI05] are designer-friendly and convenient for modellers to enhance the reconstruction results guided by strokes.

\subsection{Procedural plant modelling}

Given that tree growth is a process, most previous works considered applying procedural modelling method to simulate the growth process. This paper mainly discusses procedural modelling. 
Procedural modelling Most of the earliest research in the 1970s about plant modelling considered constructing the tree structure through a recursive procedure under some rules [Hon71]. The most famous rule-based system in this aspect is the L-system [Lin68], which many modelling methods are based from. [PL90, PHHM96] developed numerous algorithms for the L-system, which can be used to model different kinds of trees with special characteristics. Importantly, the L-system could be integrated with other modelling methods. On the basis of the L-system, Boudon et al. [BPF*03] developed an interactive modelling method, and [IOI06, OOI05] built sketch-based reconstruction modelling methods. [PHM93, PJM94] extended the L-system to a differential L-system to animate plant development, a system that defined parameters from solutions of differential equations to model the growth process of plants. Based on the L-system, [LL05, LK05] simulated the growth process under some key parameters, such as height and tree diameter at breast height (DBH), and obtained good numerical results for their models. However, these geometric models should still be developed to simulate trees.

Inverse procedural modelling Besides the L-system, some rulebased procedural methods also exist. Inverse procedural modelling methods were developed in $\left[\mathrm{SBM}^{*} 10, \mathrm{SPK}^{*} 14\right]$ to simulate the modelling or growth progress of a tree from its existing structure or from the scanned data for its structure.

Stochastic procedural modelling A plant growth modelling and simulation method based on the stochastic process, namely, AMAP, was developed in [dREF*88]. With parameters derived from observation, structures constant to botanic knowledge were modelled, where the situation of death, rest or generation of metamers of buds were concentrated. AMAP is also a type of procedural method that simulates the growth process of plants. Based on the data structure of multi-scale tree graph, a software tool AMAPstudio was proposed, which was dedicated to plant architecture modelling for botanists and agronomists [GC13], where the user may explore and edit plant topology and geometry.

Greenlab was presented as a mathematical model for plant growth based on AMAP that simulates interactions between plant structure and function [YKRD04, KCDR*08]. A new theory was proposed in [dRKHA12] to compute stochastic aspects of production, resulting from meristem extension, or rest periods and mortality. A stochastic model was developed in [KHCR12] to simulate multiple plant phenotypes with visual output. It is also proved to predict well the phenotypes of the other eight treatments (validation) through parameter interpolation. In modelling trees with Greenlab techniques, users have to specify many parameters related to measurement data; however, a practical way can extract those parameters automatically through image analysis [ICG*14].

\subsection{Modelling constrained by environmental factors}

Spatial impacts on modelling procedure Space colonization [RLP07] optimizes branch distribution and models tree traits realistically. This technique can simulate the consecutive growth process of trees. It can also generate suitable and detailed shapes of branches for different environmental conditions. [XM12, WYZB14] proposed different and improved synthetic methods based on space colonization to reconstruct and simulate tree models. Although they belong to sketch-based methods, these proposed methods express tree traits realistically, where environmental impacts on branches and interactive effects between branches are mostly considered. Particle system is used to simulate the reaction of trees under exterior effects in [PSK*12], though it is not a procedural modelling method. This system provides geometric models with suitable shapes for specific space conditions.

Modelling constrained by resources SOTM [PHL*09] utilized more botanical rules, such as branching patterns, to construct a skeleton model. This approach considers shape constraints by local branch growth and competition for lights and space. Based on the self-organization, [LRBP12] developed the algorithm into a simpler and understandable version to make it easy for modelers to design plant forms or shapes interactively.

In the LGTM approach in [YLG*15], environmental light is the main factor for branch shooting, an approach that demonstrates the sensitive property of branches influenced by others. On the basis of light information, a new resource allocation strategy is proposed based on resource models in SOTM to control plant biomorphs. The basic idea of this resource allocation model is described in Subsection 4.2, as it is one of the important bases of our new work in this paper; the other important basis is the work of [PHL*09].

In this paper, the resource allocation model of LGTM in [YLG*15] is integrated into growth equations to simulate the growth progress of plants. Compared with LGTM and those of other related models, our proposed algorithm, IGM, has three advantages. First, environmental impacts, such as space and light on the growth process, are considered. Secondly, branching algorithm is modified and applied to growth simulation, which better describes branch details and general biomorphs of a tree. Importantly, the models of our method numerically satisfy botanical constraints because of growth equations, which is useful to computer graphics and other academic research.

\section{Overview}

Terminologies and settings Some terminologies are defined in this section to clearly describe our modelling techniques:

- Node is a basic concept of the tree model; it is a point composing a branch and probably supporting stems or leaves.

- Internode is the part of a branch between two nodes.

- The length of all internodes of each tree is fixed as a constant, which is denoted as $\rho$, to simplify calculations.

- Voxel is an element of the space occupied by a tree. The space is divided into a solid grid of voxels, where the size of each voxel is set as half of $\rho$.

- Light value is associated with each voxel, and the initial light value is set as 1 .

- The direction of each shadow of a node on the environment is downward, and each light stops at the ground. This means that the sun is supposedly above the tree in our examples, as specified in Subsection 4.5. 
Pipeline Figure 1 shows the pipeline of the iteration algorithm, IGM, presented in this paper. The pipeline illustrates one circle in the entire modelling process. For simplicity, suppose that the time span of each life circle is uniform in the entire growth process.

(1) From input of the root position, the initial trunk, which consists of several nodes, is generated, or the structure data are loaded from the previous iteration.

(2) The growth resource of each node is calculated first according to light information through the resource allocation model.

(3) Results from the resource allocation model are refined by growth equation, and the fate of each existing node is determined based on refinement.

(4) Optimal branching directions and locations of newborn nodes are generated. The way of extending new nodes and branches is the same as the method in [PHL*09].

(5) The degree of impacts from newborn branches on environment is then calculated.

(6) Radii for each node are computed, and whether or not each branch is shed is calculated.

(7) After branch modelling, structure data are recorded and iteration is continued or stopped according to the growth equations and output structure data. If modelling progress stops, leaves and flowers are attached to branches after they are modelled as meshes with textures [LZCJ09].

\section{Tree Modelling Constrained by Growth Equations}

Corresponding to the above overview, the technical details of our method, IGM, are presented in this section. In the first and second subsections, we describe the modification of calculating received light resource within growth progress (Subsection 4.1) and the resource allocation model used in our algorithm (Subsection 4.2). Then, the procedure for utilizing growth equations to determine the growth in each iteration with resource distribution results is shown in Subsection 4.3. Subsections 4.4 and 4.5 explain how to append new branches and update environment information, respectively. Subsection 4.6 shows how to calculate radii and the shedding of each branch.

\subsection{Alteration of light sensitivity of a node}

In our algorithm, before the resource allocation is calculated, we need to acquire the resource value of each node by voxel light, where the node is located. When the changes of each growth are considered, light sensitivity of a node changes in simulation, while newer nodes are more light-sensitive and transmit more resource from light.

Light resource received by a node is set as $L=\alpha \cdot l^{\left(T-T_{0}\right)^{\sigma}}$, where $T$ and $T_{0}$ are the current and birth time of a node, respectively. The base of light value $l$ of a voxel is set as $l \leqslant 1$, where 1 is set for a voxel if it has full light condition. The $\sigma$ is light sensitivity of a node related to its age, where $\sigma \leqslant 1$. Figure 2 illustrates the functions of light conversion rate parameter $\alpha$ and light sensitivity parameter $\sigma$. If $\alpha$ is larger, then light energy is stronger and branches are thicker, as shown in Figure 2(a). If $\sigma$ is larger, then the nodes of tree, especially the younger nodes that are more light-sensitive
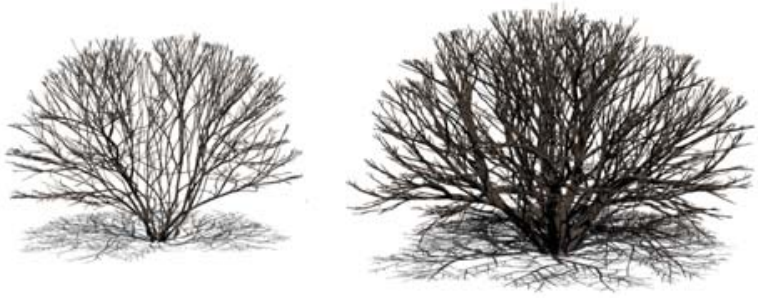

(a) Light conversion rate: $\alpha=3$ for the left and $\alpha=6$ for the right.
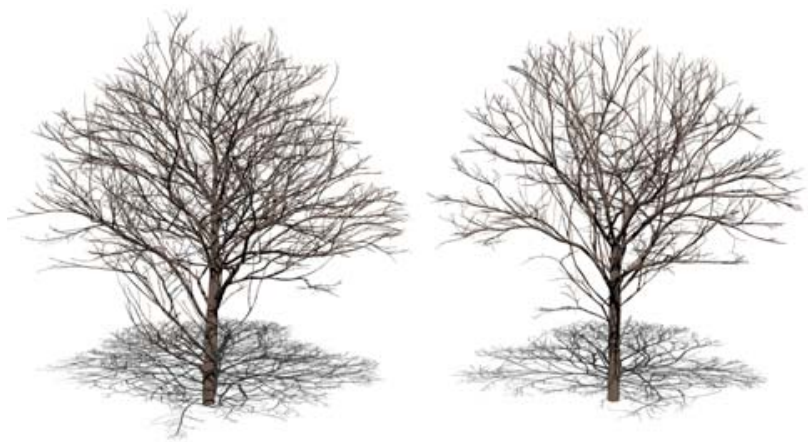

(b) Light sensitivity: $\sigma=1$ for the left and $\sigma=0.5$ for the right.

Figure 2: Influence of light parameters on tree shapes.

and receive more light energy, have higher possibility to sprout, as shown in Figure 2(b).

The light accumulation received by each node is transmitted from the top to the root and we save the total value of the whole tree at the root.

\subsection{Resource distribution}

In each iteration, the algorithm in [YLG*15] is used to calculate resource distribution. This section briefly describes this resource allocation model (for more details, see [YLG*15]). This model consists of three steps:

- First, we calculate the collected resource according to the light resource attained by each node.

- Secondly, we calculate the distributed resource for each branch to develop in the current growth period based on parameter $\lambda$, which controls the resource flow inclination to the main branches or their holding lateral branches.

- Finally, we acquire the resource attained by each node to shoot in present growth period through parameter $k$, which controls the ratio of branching nodes in an individual branch.

In addition, the apical control is considered by introducing an apical control parameter $\tau$ to generate diverse shapes. This parameter is used to control the priority of the apical node of first-level branch (trunk) when calculating its distributed resource. If $\tau=t_{1}$, the first-level branch has the apical priority from beginning to time $t_{1}$; after $t_{1}$, the priority of the apical node of trunk is equal to other nodes in the resource allocation. Moreover, if the trunk is in the period of apical control, its apical node always gets the first priority to 


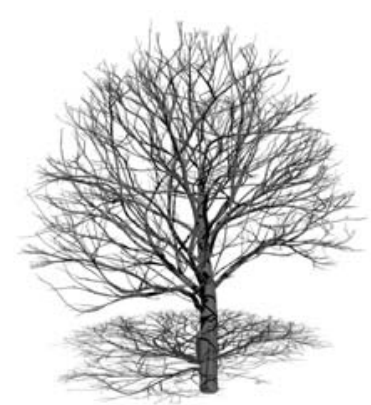

(a) $0.52 / 0.35 / 5$

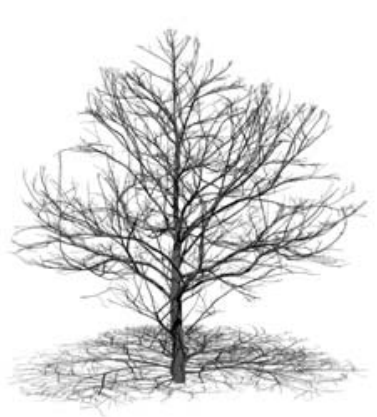

(c) $0.52 / 0.35 / 10$

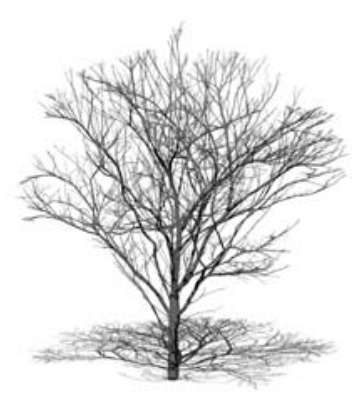

(e) $0.48 / 0.35 / 10$
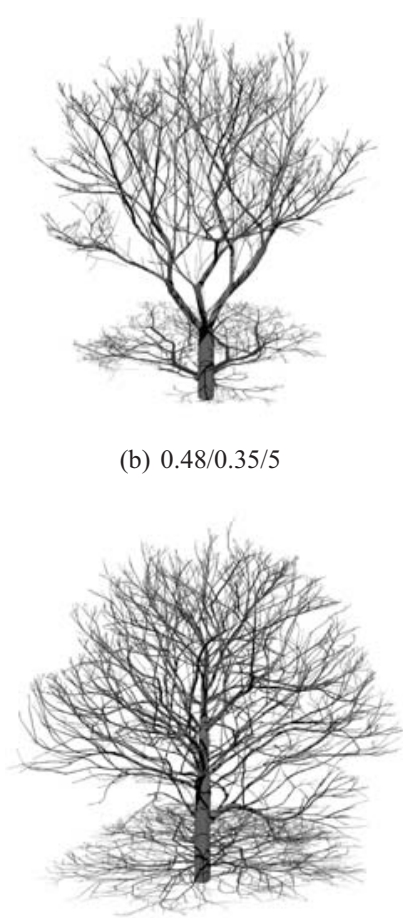

(d) $0.52 / 0.50 / 10$

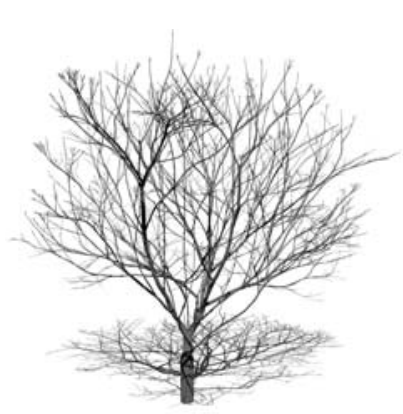

(f) $0.48 / 0.35 / 5$ (b) $0.48 / 0.35 / 5$

Figure 3: Biomorphs controlled by three parameters $\lambda, k$ and $\tau$. receive resource in the allocation phase, which means the top node of this branch gets the largest weight for receiving the distributed resource.

According to experiments, this resource allocation model is considerably useful in controlling integrated biomorphs with detailed part of models. Figure 3 shows how those parameters influence the shape of the output model. Figures 3(a) and (b) show that $\lambda$ controls the decurrent or excurrent inclination, which mainly decides the general shape. When $\lambda>0.5$, resources are more inclined to the main branches, which results in Figure 3(a) having longer and more powerful trunk and main lateral branches, while Figure 3(b) is the opposite. Figures 3(c) and (d) illustrate that if $k$ is larger, the density and strength of lateral branches increase, and vice versa. Figures 3(e) and (f) show that $\tau$, which only exists in trunk, controls the height and strength of the trunk. However, the biomorphs of trees are controlled by those parameters comprehensively so that none of the parameters has dominant effects on the final shape. Figures 3(c)-(e) show that the results of changing $\lambda$ of those two models are considerably different from the results of changing $\lambda$ of models in Figures 3(a) and (b).

\subsection{Resource constraints by growth equations}

Although botanists think that a tree has a height limitation [KSJD04, Poo99], previous published literature in the computer graphics field do not pay much attention to an algorithm on stop criterion. However, employing a growth equation not only simulates growth with variational speeds equal to a nonlinear speed curve, but also constructs a stop criterion of tree growth.

Logistic growth equation In accordance to the growth equations for plants analysed in [Zei93], we choose the logistic model [Equations (1) and (2)] proposed in 1838. Logistic equation is famous in ecology because it generally describes the increase of size of individuals or the increase of populations in a limited environment. The forces of counteraction of exponential increase are assumed to be proportional to the square of the size. Thus, logistic model is used as the main model to describe our method on how growth equations work in the algorithm. Figure 4 exhibits a virtual tree growth with the constraint of the logistic growth equation.
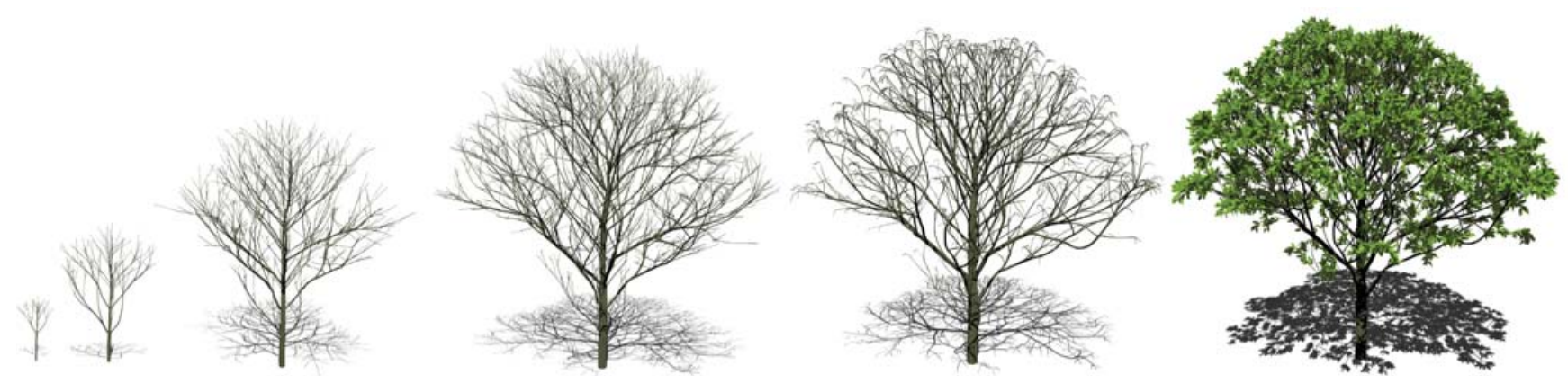

Figure 4: Logistic growth equations (1) and (2) for tree growth simulation. From left to right are models at growth circle 4, 8, 12, 16 and 20, respectively. 
Let $M$ be the maximum of node quantities from terminals to the root; then, $X_{m}=m \times \rho$. Given that the length of all internodes is set as $\rho, X_{m}$ approximates the eventual height of the model. For example, if $\rho=0.1 \mathrm{~m}$ and set $X_{m}=100 \rho$, then our method can simulate the growth of trees, which are generally $10 \mathrm{~m}$ in approximate height. The logistic equation is defined as follows:

$$
X(t)=\frac{X_{m}}{1+\left(\frac{X_{m}}{X_{0}}-1\right) \mathrm{e}^{-r t}},
$$

with its differential form (2), calculating growth speed at any time is easy.

$$
\frac{\mathrm{d} X}{\mathrm{~d} t}=X_{m} \cdot r \cdot\left(\frac{X_{m}}{X_{0}}-1\right) \mathrm{e}^{-r t} /\left(1+\left(\frac{X_{m}}{X_{0}}-1\right) \mathrm{e}^{-r t}\right)^{2}
$$

where $X_{0}$ is the initial value of $X_{m}$, and $r$ is the initial growth rate. Newborn nodes of each new shoot cannot be larger than $\frac{\mathrm{d} X}{\mathrm{~d} t}$. Parameters $X_{m}$ and $r$ can be estimated through Equation (1) by measuring real trees and employing non-linear fitting methods.

In our algorithm, the resource for growth in the present stage obtained by each node is calculated first. According to LGTM in [YLG*15], we finally attain newborn nodes $y_{n}$ from an existing node $n$ by calculating its distributed resource.

However, because of tree structure, strategy of resource distribution, and environmental effects, some nodes attain excessive resource, while trees cannot grow too much in some stages. Therefore, if a node obtains enough or excessive resource for its growth, $y_{n} \geqslant\left[\frac{\mathrm{d} X}{\mathrm{~d} t}\right]$, this node gets full growth in this period, i.e. the growth from this node calculated by the growth equation, and not by the results from the calculation of resource distribution. Conversely, if a node does not get enough resources, $y_{n}<\left[\frac{\mathrm{d} X}{\mathrm{~d} t}\right]$, newborn nodes from this node are still determined by the results from the calculation of resource distribution.

When Equation (1) is employed, the process of growth, tree height and $\mathrm{DBH}$ can be simulated in non-linear form. Figure 5 illustrates

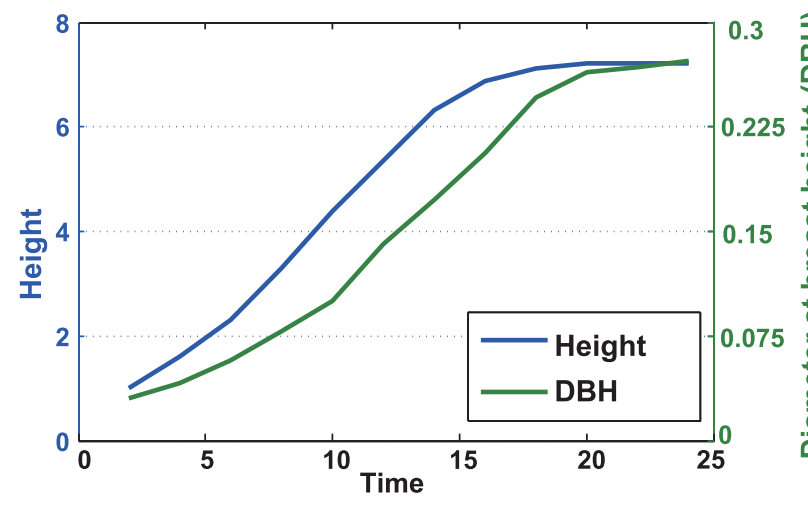

Figure 5: S-shape variation of tree heights, and DBHs, corresponding to trees in Figure 4 by logistic growth equation. the S-shape variation of tree height and DHB corresponding to the tree growth in Figure 4.

IGM without resource allocation Our method still works even without the resource allocation model, i.e. we only use growth equation to specify $y_{n}$ as $y_{n}=\left[\frac{\mathrm{d} X}{\mathrm{~d} t}\right]$. For this purpose, a simplified IGM is created on the logistic growth equation without considering resource allocations. Figure 6 shows the result of the simplified growth model. To compare the simplified model with IGM, growth parameters in Figure 6 are set the same as those in Figure 4. The two models in different figures have approximately the same size and general shape. Therefore, growth equation causes dominant effects on growth simulation.

However, the adoption of resource allocation to our method is also significant for fine details. Subsection 4.2 discusses that, with the resource allocation model, it is easy to keep the shape properties of branches, such as branch density and strength of trunk or lateral branches. Resource allocation model connects the environmental impacts on growth with the internal growth mechanism. These properties are shown in Figures 2 and 3.

Bertalanffy and Weibull equations Our algorithm also supports constraints from different growth equations. Two other growth equations, Bertalanffy and Weibull, are suitable for growth progress.

Bertalanffy equation was proposed by Bertalanffy in 1957. It 'establishes rational quantitative laws of growth and indicates the physiological mechanisms upon which growth is based' [Zei93].

$$
X(t)=\beta_{1}\left(1-\mathrm{e}^{-\beta_{2} \cdot t}\right)^{3},
$$

where $\beta_{1}$ and $\beta_{2}$ are parameters in the Bertalanffy equation.

Weibull equation is an ideal match for tree growth [ZHA97, Zei93]. Originally intended to describe a probability distribution, it has been utilized in various fields:

$$
X(t)=\omega_{1}\left(1-\mathrm{e}^{-\omega_{2} \cdot t^{\omega_{3}}}\right),
$$

where $\omega_{1}, \omega_{2}$ and $\omega_{3}$ are parameters in the Weibull equation.

Figure 6: Simplified IGM, without resource allocation model, that simulates the growth process. From left to right are models at growth circle 8,12 and 20 , respectively. 


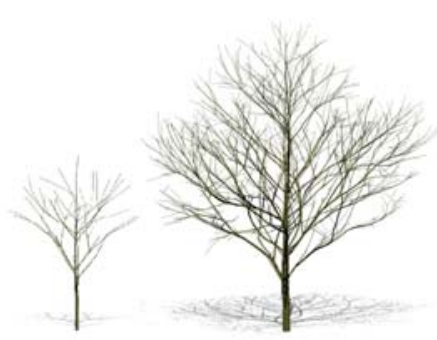

(a) Bertalanffy growth equation (3) for growth simulation.
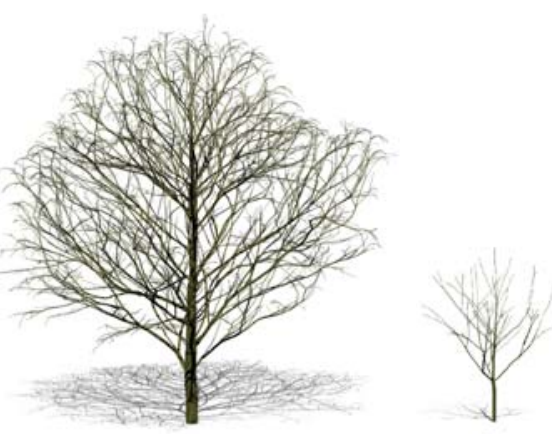
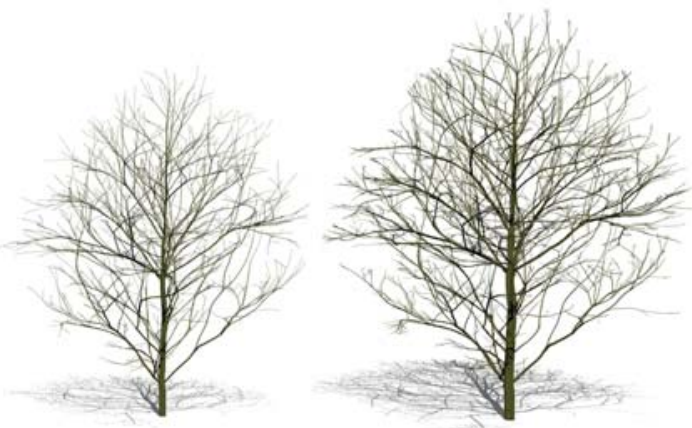

(b) Weibull growth equation (4) for growth simulation.

Figure 7: Growth simulation based on Bertalanffy and Weibull growth equations of time circle: 8, 12 and 20.

Figure 7 shows two growth models constructed, respectively, by Bertalanffy and Weibull equations. Figure 8 shows a comparison on tree height curve of the three growth equations, namely, logistic, Bertalanffy and Weibull. Therefore, modellers may choose different growth equations according to their needs to obtain a better match because our algorithm can be applied to different growth equations. In Figure 7, two models share the same parameters with that in Figure 4, except for the growth equation. Although they applied different growth equations for simulation, they still share the same configuration properties, where differences come from randomly branching directions.

Besides these three growth equations, some other growth equations [Zei93] are available to our work. Conversely, Greenlab is an advanced structural-functional model for plant growth [KCDR*08]. However, because Greenlab model is a stochastic modelling method, it is much more complex than and different from the main scheme of this work, and is thus not integrated in our paper. We will pay attention to using the structural-functional model in our future work.

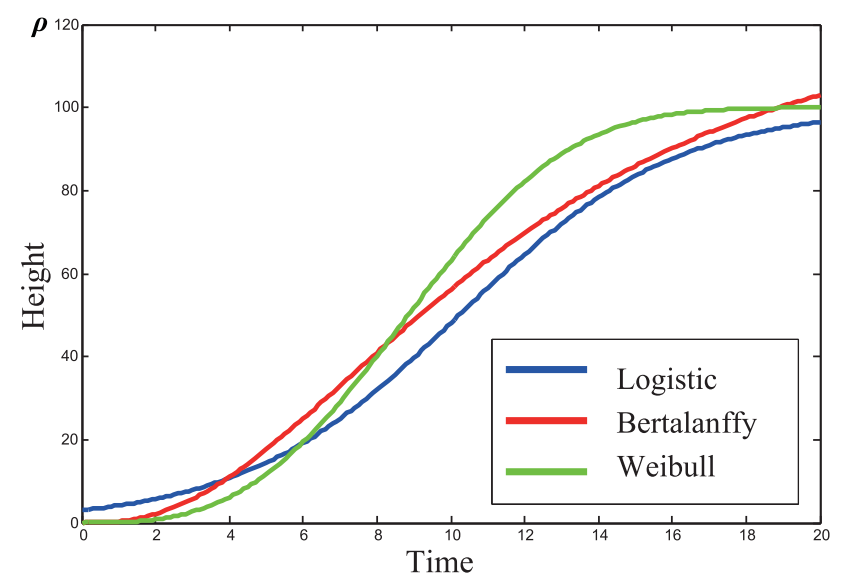

Figure 8: Curves of tree height with three growth equations, logistic, Bertalanffy and Weibull, corresponding to the trees in Figures 4 and $7(a)$ and $(b)$.

\subsection{Appendix of new shoots}

When node $i$ has the chance to extend new nodes or append new branches according to its received resource, we calculate its ultimate direction $\vec{V}_{\mathrm{u}}$ by three factors: default direction $\vec{V}_{\mathrm{d}}$, optimal direction $\vec{V}_{\mathrm{o}}$ and tropism factor $\vec{V}_{\mathrm{t}}$. Then the final shooting direction is calculated using the formula $\vec{V}_{\mathrm{u}}=\vec{V}_{\mathrm{d}}+\mu \vec{V}_{\mathrm{o}}+\gamma \vec{V}_{\mathrm{t}}$.

For $\vec{V}_{\mathrm{d}}$, if $i$ is a terminal node, $\vec{V}_{\mathrm{d}}$ is the orientation of their support internodes. If $i$ is between two internodes, $\vec{V}_{\mathrm{d}}$ is given according to phyllotaxis and the branching angle. In our experiments, branching angle is constantly initialized before the simulation.

For $\vec{V}_{\mathrm{o}}$, the modified space colonization algorithm, introduced in [RLP07, YLG*15], is utilized. At first, some random points, called marker points, are generated in a conical space around each sprouting node when the optimal direction of each node needs to be calculated. Sprouting nodes influence each other because the marker points of one node also determine others' shooting direction if they are close enough. The number of marker points of each node will decrease after each iteration, which means that the later the iteration, the greater the influence existing between the branches. Then, available marker points are determined and the optimal direction for each newborn node is calculated, as shown in Figure 9. According to our algorithm, all available points are utilized to calculate the sum of vectors from those available points to shooting nodes, and each vector has a weight equal to marker points light value according to the voxels it locates.

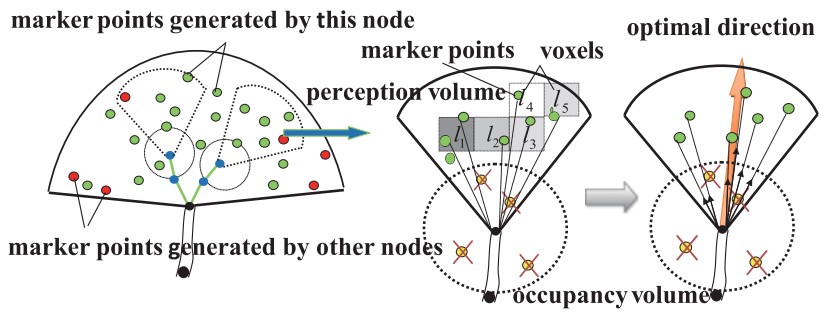

Figure 9: Steps in finding available marker points and calculating optimal direction according to light information, where $l_{j}$ is the light value of marker point $j$, and $v_{j}$ is the direction vector. 

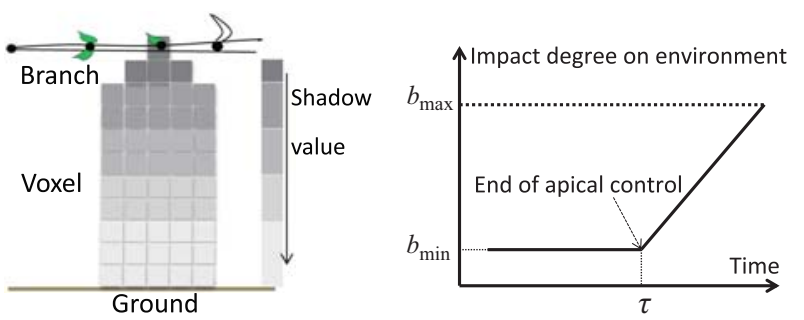

(a) Range of impact on environment.

(b) Alteration of impact parameter $b$.

Figure 10: Updating environmental light information.

Tropism factor is another factor to control the branch orientation, and its function is easy to understand. Here, we use alterable weighted tropism factor $\gamma$ in the formula to shape various trees. Figure 4 illustrates the effects if $\gamma$ is changed into a large value in the late period of growth process.

\subsection{Calculation of environmental impacts}

After generating new nodes, the impact of those nodes on the environment should be updated. The range of influence is from nodes to the ground, as illustrated in Figure 10(a), which shows a pyramid and a cube below it. For all the experiments in this paper, the depth of the inverse pyramid is 20 voxel; thus, the bottom area of the cube is 20 voxel $\times 20$ voxel. Although the influence value from one node on the ground is small, the shadow values in lower voxels are still large because of accumulation. Many of the above nodes can affect the lower voxels. Therefore, for the whole space, especially close to the tree root, the lower part will be darker.

We apply formula $l=l_{o}-b^{-q}$ to update environmental light information, where $l$ is the light value of the voxel to be updated, $l_{o}$ is the light value before the update, $b^{-q}$ is the impact value and $q$ is the number of voxels vertically between the impacted voxel and the voxel where the node belongs.

Base parameter $b$, or impact parameter, represents the impact degree of a node on the environment. Less parameter $b$ means that the node has greater influence. Figure 10(b) illustrates that parameter $b$ complies with piecewise linear function and increases in the progress, where $b_{\min }$ is the least value of $b$ that represents the greatest effects of branches on environmental light, and $b_{\max }$ is the maximum value of impact degree coefficient. Those branches generated at the early stages will be bolder than those generated at late stages, which means that they have a greater shadow impact on the environment. In our algorithm, the alteration of $b$ is set to start at the node when the apical control is dismissed at the trunk. Changing the value of $b$ and setting the impacted range makes the density of internal branches sparser than the new twigs, which will be shown in Subsection 5.4 and, more specifically, in Figure 17(b).

After updating the space information, the step appendix of new shoots is repeated until all sprouting nodes have a shot.

\subsection{Calculation of radii and shedding of branches}

The basipetal method is still used to calculate the radii of branches by using formula $R^{v}=\sum_{j=1}^{N} R_{j}^{v}$, where $R$ is the radius of this internode, $R_{j}$ is the radii of the children internodes of this internode, $N$ is the total number of successors, including the main axis and lateral branches and $v$ is a user-defined parameter related to tree species. Moreover, radii will not decrease by the shedding of branches. After each iteration, the branch shedding step is applied as [Tak94]. For each branch, the total amount of light it received is compared with the branch size measured in the number of internodes.

\section{Experimental Results}

All the results presented in this paper were conducted on a PC with 2.4 GHz Q2600 Quad CPU, 16 GB memory, and a 64-bit Windows 7 operating system.

\subsection{Modelling constrained by solid obstacles}

Our method is capable of simulating the growth process close to some obstacles. The main idea is different from the deformation of a mature tree, i.e. to build a new structure when a tree meets a solid obstacle [PSK*12]. Hence, our method is considered to exhibit the growth process, though it does not increase the complexity of our algorithm.

Figure 11 simulates a tree growing close to a wall, while Figure 12 simulates a tree growth under a solid thin board, where the grey board exists in the upperleft part of the picture. Simulation experiments show that branches averted growing too much in shadow space and circumvented the obstacles to expand their colonization. Meanwhile, the whole tree adjusted its resource allocation according to the environment. Although resources are also allocated much more to the part of the tree with more light, it still has constraints to the growth.

\subsection{Modelling in a dynamic environment}

The growth of multiple trees can be simulated with our algorithm, which can be expanded only by setting the tree number and their root positions, and initializing the light covering all the tree growth environment. In our experiments, received resources by the growing trees are influenced by one another. In each iteration, the growth of all trees should be calculated before the appendix of new branches of different individuals. The order of calculation of growth is randomly decided in each iteration. By calculating environmental constraints, branches from different trees will not overlap.

Figure 13 exhibits a situation of five trees growing simultaneously close to one another. Central tree (b) and one of the peripheral trees (c) show the mutual effects of the models on the environment, as well as the space competition between models. Moreover, the top view of models (d), show the colonized space of each tree, which proves that our algorithm can model tree growth in a complex environment, and that its results satisfy realistic situations. 

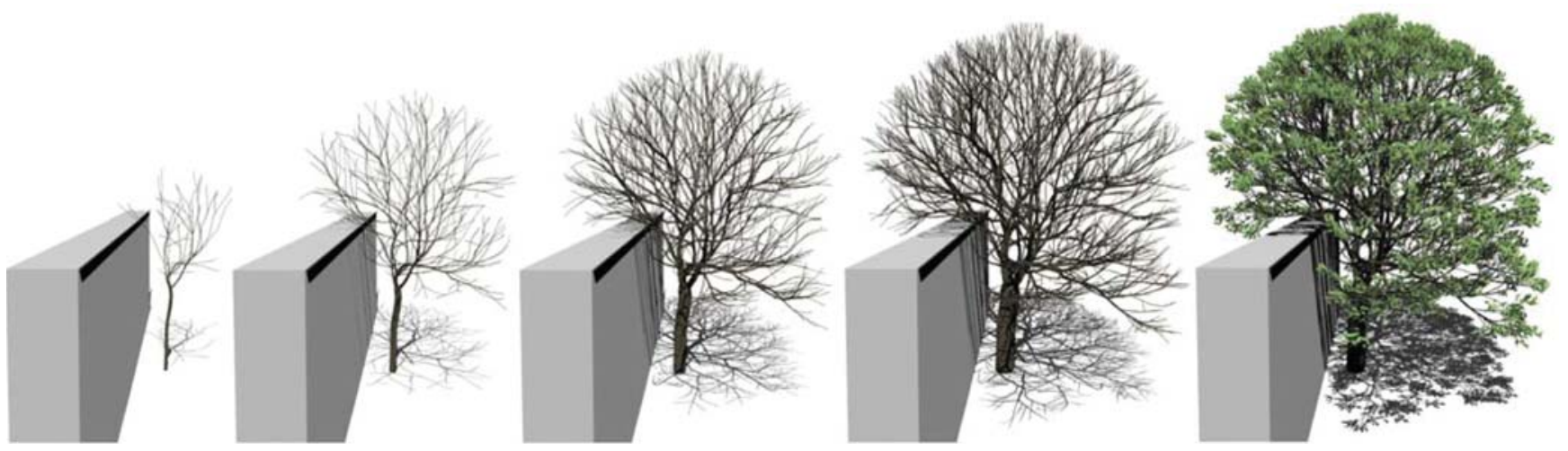

Figure 11: Growth simulation with wall occlusion.
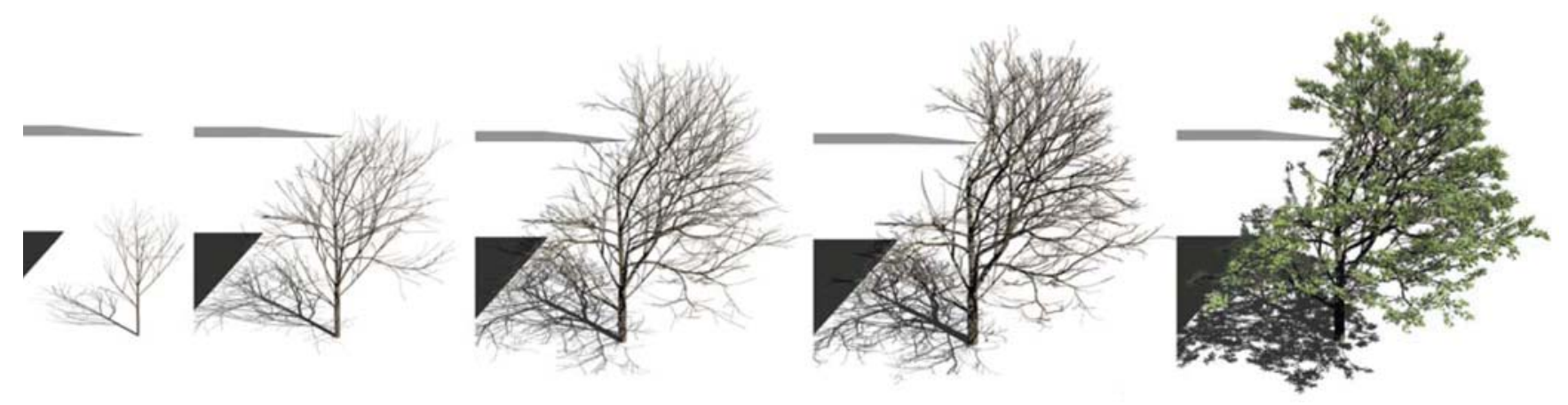

Figure 12: Growth simulation of trees with a solid thin board.

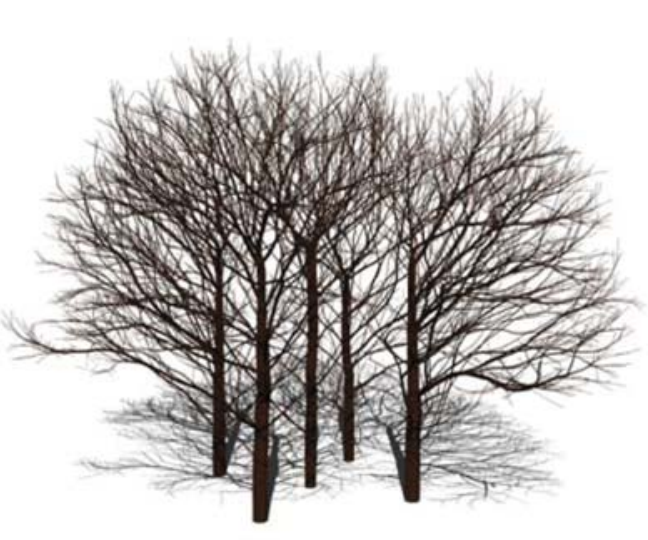

(a)

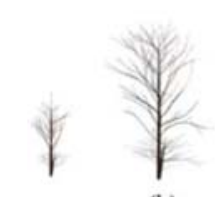

(b)
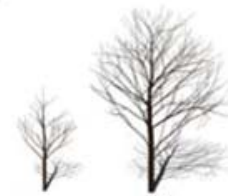

(c)
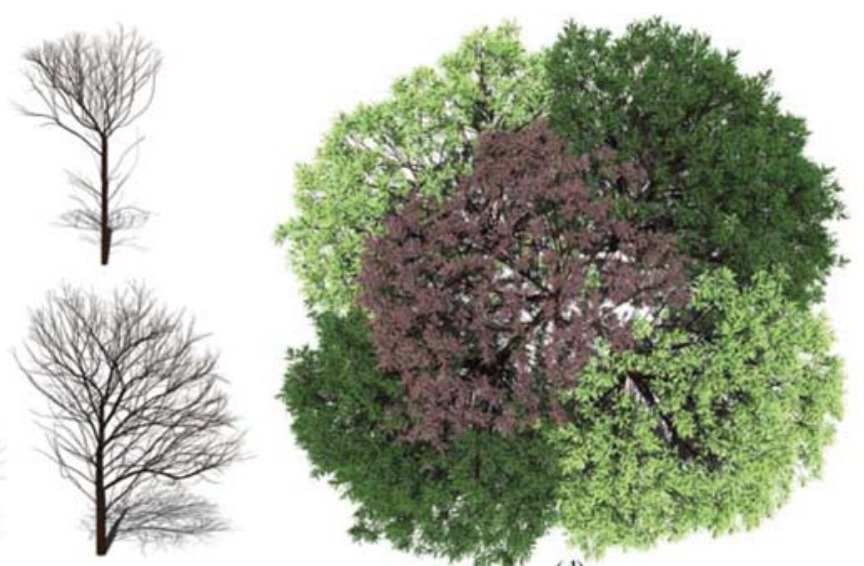

(d)

Figure 13: Growth simulation of the five trees. These trees are generated based on the same parameters and are shown in different colours for easy recognition. (a) Front view of the five trees; (b) Growth of the central tree; (c) One of the peripheral trees that grow more than the central tree (b). This figure shows that the trees generated by our method exhibit individually different shapes according to different environmental conditions. (d) Top view of trees.

\subsection{Shrub modelling}

By setting several roots closely and with limited tree height, our method can generate shrub plants. We suppose that the resources from ground are infinite, and the different roots of plants will not compete for resource underground. According to the shape of real Amygdalus triloba, we specify the parameters to construct a model shown in Figure $14: X_{m}=50 \rho, \lambda=0.46, k=0.5$ and $\mu=0.6$. 


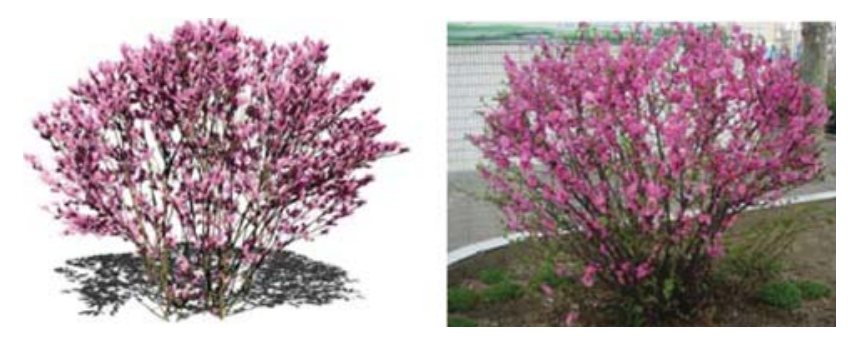

Figure 14: Simulation of the growth of two trees competing for space and resources with different growth equations based on our method.

\subsection{Comparisons with related works}

The growth process of two or more plants with different biomorphs in a competitive environment can be simulated by our method.

Figure 15 simulates two plant growths closely. The growth speed of the shorter plant decreased gradually because it lost the competition with the higher one. Logistic equation is still utilized to simulate the process. First, the growth equation of the left plant with $X_{m}=70 \rho$ is slower than the right with $X_{m}=100 \rho$. Thus in the following simulation, the left one lost the competition for light resource and gradually stopped growing in the late stages, where the resource allocation model dominates the growth size. Consequently, the left one cannot get enough resource to grow into the height it should have. However, the final result of the simulation of two trees in Figure 15 is determined not only by growth equations, but also by a comprehensive control of all the parameters, such as $\lambda, k, \tau, \alpha, \sigma, \mu, \gamma$ and $b$, which are listed in Table 2. To make the simulation closer to the real growth process, we have also proposed some other modifications in our algorithm above. Light sensitivity (with parameters $\alpha$ and $\sigma$ ) of each node alters during growth simulation.

To compare with our method, SOTM, which was proposed by [PHL*09], is reproduced to simulate the same situations. SOTM is also a modelling method based on resource distribution. The models likewise share the same parameters compared with models in Figure 15 constructed in our method. Figure 16 shows that the two models almost have same growth in each stage, while having obviously different growth in the last three stages in the growth sequence from our method. Moreover, the dominant effect of the growth equation on the biomorph of models can be seen from the comparison. Therefore, it is convenient for modellers to change the species or general shape of tree models by just tuning the parameters of the growth equation.

Compared with the method in [YLG*15], our algorithm can control branch density, such that the simulation satisfies the property of variant branch density, because our method gradually alters light sensitivity of nodes and impacts parameter $b$, which represents the impact of a node on the environment, while the method in [YLG*15] cannot. Figure 17 shows a comparison of branch density in the interior part of the trees, in which the variation of branch density is clear through the zoomed in part of pictures in the left part of Figures 17(a) and (b). In nature, some tree species make their branches located at the inner or lower part hold sparser density than those in the exterior or upper part, as shown in Figure 17(c).

The general advantages of our method compared with the method in [YLG*15, PHL*09] are listed in Table 1 , where

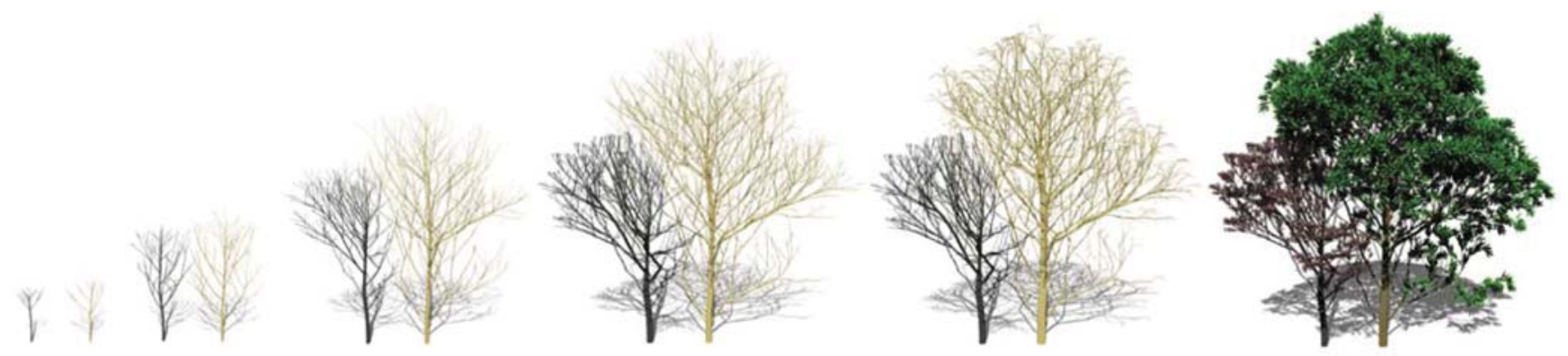

Figure 15: Simulation of the growth of two trees competing for space and resources without growth equations based on SOTM [PHL*09].
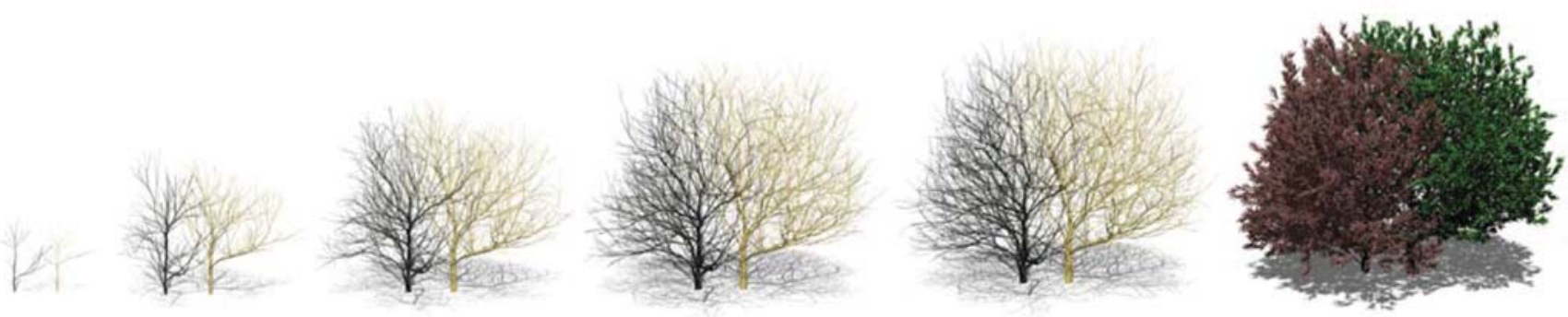

Figure 16: Modelling a shrub. (Left) Amygdalus triloba model through our method; (Right) photo of the shrub. 


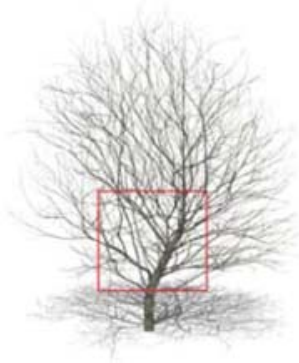

(a) LGTM in $\left[\mathrm{YLG}^{*} 15\right]$

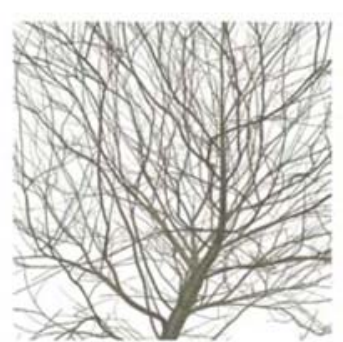

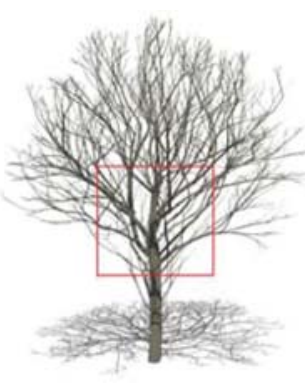

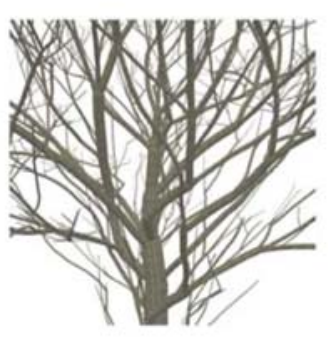

(b) Ours (IGM).

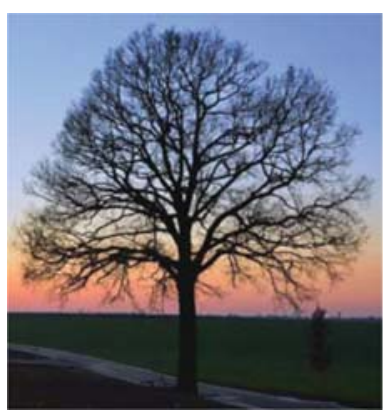

(c) The photo of a real tree.

Figure 17: Comparison of branch density in the interior part of trees. (a) Tree model constructed by [YLG*15]; (b) Tree constructed by our model; (c) Photo of a real tree.

Table 1: Comparison with related work.

\begin{tabular}{llllllll}
\hline Method & Resource Alc. & Envir. & Equation & Growth & Branch density & Stopping criteria & Height, DBH \\
\hline SOTM [PHL*09] & BH or Priority & Yes & No & No & Self-organized & Resource & Not mention \\
LGTM [YLG*15] & BH + Priority & Yes & No & No & Light & Resource & Not mention \\
Ours (IGM) & BH + Priority & Yes & Yes & Yes & Integrated factors & Resource+ Equation & Yes \\
\hline
\end{tabular}

- Resource Alc. means the strategy chosen for resource allocation used in the algorithm. BH means Borchert-Honda model, while Priority means priority model. Different references realize these two allocation strategies differently or modify some parts of them. However, the basic ideas are based on these two models.

- Envir. means whether the method considered the environmental impacts.

- Equation means whether the method is capable of simulating the growth process constrained by growth equations.

- Growth means whether the method concentrates or emphasizes the simulation of growth process.

- Branch density means the way of controlling the branch density of the tree model for modellers or for the method itself. Our method considered numerous factors integrated into the growth process introduced before.

- Stopping Criteria means the criterion of terminating the simulation process.

- Height, $D B H$ means whether the method numerically perform height/DBH analysis in the botanic aspect. Here, the compared papers do not mention the analysis about this aspect.

Only the parameters involved in our algorithm are listed here because we compare with different algorithms. Given that the approaches in [YLG*15] and [PHL*09] do not have stopping criteria, they are assigned the same times of growth iterations as the models of our method.

\subsection{Diverse tree biomorphs and control parameters}

We list more experimental results by tuning several parameters with our method. Figure 18 shows three trees with different architecture shapes: a broad-leaved tree, an evergreen shrub and a cypress tree. The parameters listed in Table 2 are used to generate all trees in Section 5, and some information is shown on these tree models. In the table, $r_{\mathrm{s}}$ means the ratio of shedding according to [Tak94]; $H$ and $D B H$, respectively, mean the final trees' height and $\mathrm{DBH}$ and $L o S$ is the loop of the model finishing simulation, which means that after $L o S$ loops, the tree stops growing. Please note that given the same set of parameters for a specific tree type, our algorithm will generate trees with similar height and crown shapes but the geometry is not exactly the same, because we generate random marker points around each bud when shooting new branches.

The processes to simulate shrubs also started with several roots, as described in Subsection 5.3. Therefore, the tree in Figure 14 starts the process with 10 roots, and the second tree in Figure 18 has six roots at the beginning of the simulation.

\subsection{Strategy for tuning parameters}

This section introduces how to tune the main parameters. According to Section 4, every parameter listed in Table 2 controls some aspects of the shape. Although most of the parameters can be set as constant and do not need to tune too much, it is still hard to tune the parameters integrally without deep understanding of parameters' functions. Thus, we introduce some simple strategies to help reader reproduce the results presented in this paper. Fortunately, according to our practical experience, we just need to tune 5 main parameters to generate the growth process of different kind of plants. In Table 3, we show the typical range of these parameters and the modelling effects when setting a higher value for each of them. 
Table 2: Parameter setting and tree information in Section 5.

\begin{tabular}{|c|c|c|c|c|c|c|c|c|c|c|c|c|c|c|}
\hline Tree & $\lambda$ & $k$ & $\tau$ & $\rho$ & $X_{0}, X_{m}, r$ & $\alpha$ & $\sigma$ & $\mu$ & $\gamma$ & $b_{\min }, b_{\max }$ & $r_{\mathrm{s}}$ & $H$ & $\mathrm{DBH}$ & LoS \\
\hline Figure 11 & 0.52 & 0.5 & 6 & 0.10 & $2,100,0.34$ & 4 & 0.5 & 1.5 & 0.5 & $1.8,2.2$ & 0.25 & 6.09 & 0.22 & 20 \\
\hline Figure 12 & 0.54 & 0.35 & 6 & 0.10 & $2,100,0.34$ & 4 & 0.5 & 1.5 & 0.5 & $1.8,2.2$ & 0.35 & 7.45 & 0.26 & 20 \\
\hline Figure 13(b) & 0.52 & 0.5 & 5 & 0.10 & $2,100,0.34$ & 4 & 0.5 & 1.5 & 0.5 & $1.8,2.3$ & 0.35 & 8.57 & 0.23 & 20 \\
\hline Figure 13(c) & 0.52 & 0.5 & 5 & 0.10 & $2,100,0.34$ & 4 & 0.5 & 1.5 & 0.5 & $1.8,2.3$ & 0.35 & 7.95 & 0.27 & 20 \\
\hline Figure 15, left & 0.48 & 0.5 & 4 & 0.10 & $2,70,0.34$ & 3 & 0.5 & 1.5 & 0.5 & $1.8,2.5$ & 0.20 & 5.23 & 0.17 & 14 \\
\hline Figure 15 , right & 0.52 & 0.5 & 4 & 0.10 & $2,100,0.34$ & 4 & 0.5 & 1.0 & 0.2 & $1.8,2.2$ & 0.35 & 8.07 & 0.22 & 20 \\
\hline Figure 16, left & 0.48 & & 4 & 0.10 & & & & 1.5 & 0.2 & $1.8,2.5$ & 0.50 & 7.72 & 0.20 & \\
\hline Figure 16, right & 0.52 & & 4 & 0.10 & & & & 1.0 & 0.2 & $1.8,2.2$ & 0.35 & 8.60 & 0.28 & \\
\hline Figure 14 & 0.46 & 0.5 & 0 & 0.02 & $2,50,0.34$ & 6 & 1.0 & 0.6 & 1.0 & $2.0,2.5$ & 0.15 & 0.68 & & 10 \\
\hline Figure 17(a) & 0.52 & 0.5 & 6 & 0.10 & & & & 1.0 & 0.5 & & 0.35 & 9.59 & 0.31 & \\
\hline Figure 17(b) & 0.52 & 0.5 & 6 & 0.10 & $2,100,0.34$ & 4 & 0.5 & 1.0 & 0.5 & $1.8,2.2$ & 0.35 & 7.22 & 0.28 & 20 \\
\hline Figure 18 , first & 0.48 & 0.5 & 2 & 0.10 & $2,100,0.34$ & 6 & 0.5 & 1.0 & 0.5 & $1.8,2.5$ & 0.25 & 5.21 & 0.13 & 20 \\
\hline Figure 18, second & 0.46 & 0.5 & 0 & 0.01 & $2,50,0.34$ & 6 & 1.0 & 1.0 & 0.2 & $2.0,2.5$ & 0.05 & 0.29 & & 12 \\
\hline Figure 18, third & 0.56 & 0.5 & 14 & 0.20 & $2,100,0.34$ & 3 & 0.5 & 0.5 & 1.5 & $1.8,2.2$ & 0.45 & 8.06 & 0.35 & 20 \\
\hline
\end{tabular}
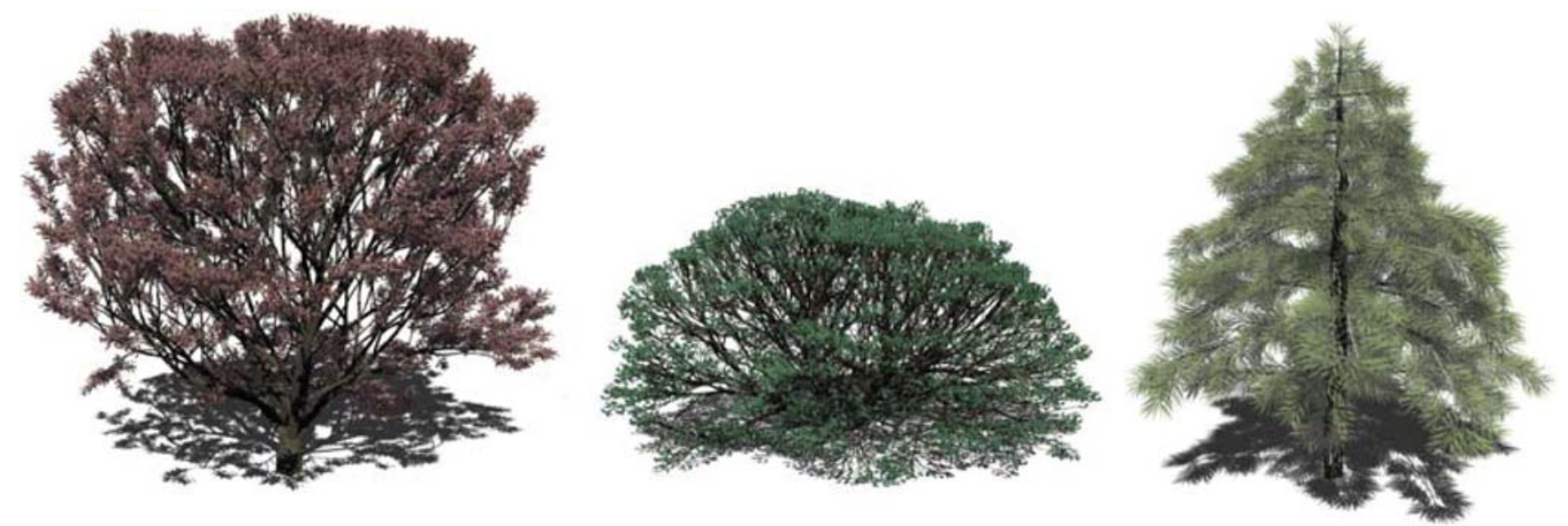

Figure 18: Tree models with different architecture shapes: a broad leaved tree, an evergreen shrub and a cypress tree.

Table 3: The main parameters for simply tuning.

\begin{tabular}{lcl}
\hline Paras. & Range & \multicolumn{1}{c}{ The effects of higher value } \\
\hline$\lambda$ & $0.45-0.55$ & More lateral branches \\
$\tau$ & $0-15$ & Longer and stronger main trunk \\
$X_{m}$ & $50-200$ & Larger size and higher height \\
$\gamma$ & $0-2$ & More gravity influence on branches growth \\
$r_{\mathrm{s}}$ & $0.15-0.45$ & Higher density of branches/more prosperous \\
\hline
\end{tabular}

\section{Conclusion and Discussion}

A new generic tree modelling method that considers growth factors, i.e. light resource, space occupation and environmental impacts, is proposed to model virtual trees and simulate their growth. Specifically, these factors are models as a set of parameters in the growth equations that controls the tree growth. Our main technical contributions are as following:
- An integration of growth equations with the procedural modelling. Our tree modelling approach integrates growth equation into the procedural modelling of trees. The whole shape modelling procedure is a list of growth series of trees, satisfying a mathematical equation of the growth. Therefore, we apply the growth equation to refine every iteration of procedural tree modelling. Given that the simulation is constrained by a mathematical equation, our method numerically satisfies botanical constraints stated in citeZeide93, which is useful to plant-related academic research, like botany, forestry and agriculture.

- Environmental impacts and resource allocation. The introduction of growth equations provides a new mechanism to simulate tree growth within environmental impacts and resource allocation. Our algorithm can automatically adjust the degree of mutual influences of trees and their environment, and the mode of resource allocation according to different growth periods.

The technical advantages over state-of-the-art approaches are as following: 
- Height limitation. The advantage of height limitation of the tree growth simulation is due to the growth equation as dominant constraints on tree modelling; even the number of algorithm iteration is sufficiently great [KSJD04, Poo99].

- Mutual influences. Our method concentrates on growth simulation in restrictive or competitive environments based on growth equation, where mutual influences of individual trees are illustrated.

- Bush modelling. By setting the number of roots, start positions and initial light space, bushes can be modelled using our method.

- Variant branch density. Our method provides a way to simulate variant branch density in the crown of a tree, which is a shape property of some trees, because the impact of a tree on the environment alters according to the growth process.

Our method still has limitations. (1) The influence of gravity to branching is not considered, resulting in the shape of some branches not simulated well. (2) Several parameters for modelling must be tuned, which can be ameliorated by building a simplified simulation model with a strong control of morphological traits. Furthermore, the parameters are only specified by the user and should thus be calibrated by real measurement data. (3) Mathematic equations are used only to control growth speed. Age circles and the growth of organs, such as leaves, flowers and fruits, are not considered.

Therefore, we should investigate some valuable aspects in the future. Four aspects should be considered: (1) the impact of gravity on growth process; (2) inverse simulation and numerical analysis of the growth process of some grown trees according to growth equations; (3) using image and point cloud data to estimate parameters in growth equations and (4) employing structural-functional models, like Greenlab, to simulate organ growth and dry matter flow, as well as analyse the resource acquirement and allocation.

\section{Acknowledgements}

We would like to thank the reviewers for their thoughtful comments. This work is supported in part by National Natural Science Foundation of China with Nos. 61331018, 61372190, 61571439, 61571400 , in part by the National High Technology Research and Development Program of China (2015AA016402), in part by the National Foreign 1000 Talent Plan (WQ201344000169) and in part by Leading Talents of Guangdong Program (00201509).

\section{References}

[BNB13] Bradley D., Nowrouzezahrai D., Beardsley P.: Imagebased reconstruction and synthesis of dense foliage. ACM Transactions on Graphics (TOG) 32, 4 (2013), 74:1-74:10.

[BPF*03] Boudon F., Prusinkiewicz P., Federl P., Godin C., KarwowskI R.: Interactive design of bonsai tree models. Computer Graphics Forum 22, 3 (2003), 591-599.

[CNX*08] Chen X., Neubert B., Xu Y. Q., Deussen O., Kang S. B.: Sketch-based tree modeling using Markov random field. ACM Transactions on Graphics 27, 5 (2008), 1-9.
[DL06] Deussen O., Lintermann B.: Digital Design of Nature: Computer Generated Plants and Organics. Springer Science \& Business Media, 2006.

[dREF*88] de RefFye P., Edelin C., Françon J., Jaeger M., Puech C.: Plant models faithful to botanical structure and development. In SIGGRAPH '88: Proceedings of the 15th Annual Conference on Computer Graphics and Interactive Techniques (New York, NY, USA, 1998), ACM, pp. 151-158.

[dRKHA12] de Reffye P., Kang M., Hua J., Auclair D.: Stochastic modelling of tree annual shoot dynamics. Annals of Forest Science 69, 2 (2012), 153-165.

[GC13] Griffon S., Coligny F. D.: AMAPstudio: An editing and simulation software suite for plants architecture modelling. Ecological Modelling 290 (2013), 3-10.

[Hon71] Honda H.: Description of the form of trees by the parameters of the tree-like body: Effects of the branching angle and the branch length on the shape of the tree-like body. Journal of Theoretical Biology 31, 2 (1971), 331-338.

[ICG*14] Iovan C., Cournède P. H., Guyard T., Bayol B., Boldo D., CORD M.: Model-based analysis-synthesis for realistic tree reconstruction and growth simulation. IEEE Transactions on Geoscience and Remote Sensing 52, 2 (Feb. 2014), 1438-1450.

[IOI06] IJIRI T., OwADA S., IgARASHI T.: The Sketch L-System: Global Control of Tree Modeling Using Free-Form Strokes. Springer Berlin Heidelberg, 2006, pp. 138-146.

[KCDR*08] Kang M. Z., Cournède P. H., De RefFye P., Auclair D., Hu B. G.: Analytical study of a stochastic plant growth model: Application to the greenlab model. Mathematics and Computers in Simulation 78, 1 (2008), 57-75.

[KHCR12] Kang M. Z., Heuvelink E., Carvalho S. M. P., Reffye P. D.: A virtual plant that responds to the environment like a real one: The case for chrysanthemum. New Phytologist 195, 2 (2012), 384-395.

[KSJD04] Koсh, G. W., Sillett S. C., Jennings G. M., Davis S. D.: The limits to tree height. Nature 428, 6985 (2004), 851-854.

[LDY10] Lopez L. D., Ding Y., Yu J.: Modeling complex unfoliaged trees from a sparse set of images. Computer Graphics Forum 29, 7 (2010), 2075-2082.

[Lin68] LindenMAYER A.: Mathematical models for cellular interactions in development. II. Simple and branching filaments with two-sided inputs. Journal of Theoretical Biology 18 (1968), 300315.

[LK05] Lam Z., KING S. A.: Simulating tree growth based on internal and environmental factors. In GRAPHITE '05: Proceedings of the 3rd International Conference on Computer Graphics \& Interactive Tech (2005), ACM, pp. 99-107.

[LL05] Linsen L., Karis B. J., Mcpherson E. G., Hamann B.: Tree growth visualization. Journal of WSCG 13 (2005), 81-88. 
[LPC*11] Livny Y., Pirk S., Cheng Z., Yan F., Deussen O., CohenOr D., Chen B.: Texture-lobes for tree modeling. ACM Transactions on Graphics 30, 4 (July 2011), 53:1-53:10.

[LRBP12] Longay S., Runions A., Boudon F., Prusinkiewicz P.: Treesketch: Interactive procedural modeling of trees on a tablet. In SBIM '12: Proceedings of the International Symposium on Sketch-Based Interfaces and Modeling (Aire-la-Ville, Switzerland, 2012), Annecy, France, pp. 107-120.

[LYO*10] Livny Y., Yan F., Olson M., Chen B., Zhang H., El-Sana J.: Automatic reconstruction of tree skeletal structures from point clouds. ACM Transactions on Graphics 29, 6 (2010), 151:1151:8.

[LZCJ09] Li H., Zhang X., Che W., JAeger M.: Smooth transition between different plant leaves models. In Proceedings of 2009 Third International Symposium on Plant Growth Modeling, Simulation, Visualization and Applications (PMA) (Beijing, China, 2009), IEEE, pp. 376-383.

[NFD07] Neubert B., Franken T., Deussen O.: Approximate imagebased tree-modeling using particle flows. ACM Transactions on Graphics 26, 3 (July 2007), 88:1-88:8.

[OOI05] Okabe M., Owada S., Igarash T.: Interactive design of botanical trees using freehand sketches and example-based editing. Computer Graphics Forum 24, 3 (2005), 487-496.

[PHHM96] Prusinkiewicz P., Hammel M., Hanan J., Mech R.: Lsystems: From the theory to visual models of plants. In Proceedings of the 2nd CSIRO Symposium on Computational Challenges in Life Sciences (1996), vol. 3, Citeseer, pp. 1-32.

[PHL*09] Palubicki W., Horel K., Longay S., Runions A., Lane B., Mech R., Prusinkiewicz P.: Self-organizing tree models for image synthesis. ACM Transactions on Graphics 28, 3 (2009), 58:1-58:10.

[PHM93] Prusinkiewicz P., Hammel M. S., Muolsness E.: Animation of plant development. In SIGGRAPH '93: Proceedings of the 20th Annual Conference on Computer Graphics and Interactive Techniques (New York, USA, 1993), ACM, pp. 351-360.

[PJM94] Prusinkiewicz P., James M., MĚch R.: Synthetic topiary. In SIGGRAPH '94: Proceedings of the 21st Annual Conference on Computer Graphics and Interactive Techniques (New York, USA, 1994), ACM, pp. 351-358.

[PL90] Prusinkiewicz P., Lindenmayer A.: The Algorithmic Beauty of Plants. (New York, USA, 1990), Springer-Verlag New York, Inc.

[Poo99] PoORTER L.: Growth responses of 15 rain-forest tree species to a light gradient: The relative importance of morphological and physiological traits. Functional Ecology 13, 3 (1999), 396-410.

[PSK*12] Pirk S., Stava O., Kratt J., Said M. A. M., Neubert B., Měch R., Benes B., Deussen O.: Plastic trees: Interactive self-adapting botanical tree models. ACM Transactions on Graphics 31, 4 (July 2012), 50:1-50:10.
[QTZ*06] Quan L., Tan P., Zeng G., Yuan L., Wang J., Kang S. B.: Image-based plant modeling. ACM Transactions on Graphics (TOG) 25, 3 (2006), 599-604.

[RCSL03] Rodkaew Y., Chongstitvatana P., Siripant S., LuRsinsaP C.: Particle systems for plant modeling. Plant Growth Modeling and Applications (2003), 210-217.

[RLP07] Runions A., Lane B., PrusinKIEwicz P.: Modeling trees with a space colonization algorithm. In NPH '07: Proceedings of the Third Eurographics Conference on Natural Phenomena (Airela-Ville, Switzerland, 2007), Eurographics Association, pp. 6370.

[SBM*10] Stava O., Benes B., Měch R., Aliaga D. G., Kristof P.: Inverse procedural modeling by automatic generation of Lsystems. Computer Graphics Forum 29, 2 (2010), 665-674.

[SPK*14] Stava O., Pirk S., Kratt J., Chen B., Mech R., Deussen O., Benes B.: Inverse procedural modelling of trees. Computer Graphics Forum 33, 6 (2014), 118-131.

[Tak94] TAKENAKA A.: A simulation model of tree architecture development based on growth response to local light environment. Journal of Plant Research 107, 3 (1994), 321-330.

[TFX*08] Tan P., Fang T., Xiao J., Zhao P., Quan L.: Single image tree modeling. ACM Transactions on Graphics 27, 5 (2008), 108:1-108:7.

[WBCG09] Wither J., Boudon F., CANi M.-P., Godin C.: Structure from silhouettes: A new paradigm for fast sketch-based design of trees. Computer Graphics Forum 28, 2 (2009), 541-550.

[WP95] Weber J., Penn J.: Creation and rendering of realistic trees. In SIGGRAPH '95: Proceedings of the 22nd annual conference on Computer Graphics and Interactive Techniques (New York, USA, 1995), ACM, pp. 119-128.

[WYZB14] WANG R., YANG Y., Zhang H., BAO H.: Variational tree synthesis. Computer Graphics Forum 33, 8 (2014), 82-94.

[XGC07] Xu H., Gossett N., Chen B.: Knowledge and heuristicbased modeling of laser-scanned trees. ACM Transactions on Graphics 26, 4 (2007), 19:1-19:13.

[XM12] Xu L., Mould D.: Synthetic tree models from iterated discrete graphs. In GI '12: Proceedings of Graphics Interface 2012 (Toronto, Canada, 2012), Canadian Information Processing Society, pp. 149-156.

[YKRD04] Yan H.-P., Kang M. Z., RefFye P. D., Dingkuhn M.: A dynamic, architectural plant model simulating resourcedependent growth. Annals of Botany 93, 5 (2004), 591-602.

[YLG*15] Yi L., Li H., Guo J., Deussen O., Zhang X.: Lightguided tree modeling of diverse biomorphs. In Pacific Graphics Short Papers (2015), Stam J., Mitra N. J., Xu K. (Eds.), The Eurographics Association.

[Zei93] ZeIDE B.: Analysis of growth equations. Forest Science 39, 3 (Aug. 1993), 594-616. 
[ZHA97] ZHANG L.: Cross-validation of non-linear growth functions for modelling tree height-diameter relationships. Annals of Botany 79, 3 (1997), 251-257.

[ZLD*14] Zhang X., Li H., Dai M., MA W., Quan L.: Data-driven synthetic modeling of trees. IEEE Transactions on Visualization and Computer Graphics 20, 9 (2014), 1214-1226.

\section{Supporting Information}

Additional Supporting Information may be found in the online version of this article at the publisher's web site:

\section{Video S1}

